\title{
Los trabajos tutorizados como método de aprendizaje a través de la investigación y la aplicación de las nuevas tecnologías
}

\author{
Francisco Martínez González \\ Universidad de Mayores. Universidad Politécnica de Cartagena, España \\ f.martínez@upct.es
}

\begin{abstract}
The Technical University of Cartagena in the 2008/2009 academic course launched in the field of the Senior Citizens University, the educational project Trabajos Tutorizados (TT coursework tutored), which has been offered to all students who have completed the training itinerary that forms our University Program for the elderly. We look at the formative action TT, as the activity which, like the end of degree work or master, mark the culmination of the University career of the student. In these, under the supervision of a Tutor, is intended that students faced with a task of exceeding the developed complexity throughout his tenure in university classrooms, inviting him to undertake a bibliographic study, to apply the acquired knowledge to bring into play the talents of creativity and originality, to obtain results and to quantify its effort. Students receive prior training through the course of introduction research in science and technology, theoretical-practical in nature, with duration of 30 hours. This course, in addition to introducing conceptual aspects of research in these areas and its methodology, addresses the use of new technologies at different stages of research: update of information, the processing of the data or the disclosure of the results obtained. The use of databases, the processing of data through Microsoft Excel $^{\mathrm{mm}}$ or the presentation of the results using Microsoft Power Point ${ }^{\mathrm{m} \text {, }}$, are some of the tools of ICT, students learn to use. The History of Science and Technology, Mathematics, Astronomy or Biology, are some of the fields in which our students have done тт. The student develops a Final Memory of his work and a poster of the same. An exhibition of posters of the lockout work is carried out at the end of the course.
\end{abstract}

Keywords: University Programs for the Elderly, dissemination of Science and Technology, ICT and the elderly

\section{Resumen}

La Universidad Politécnica de Cartagena en el curso académico 2008/2009 puso en marcha, en el ámbito de la Universidad de Mayores, el proyecto educativo Trabajos Tutorizados (тт), que se oferta a todos los alumnos que han completado el itinerario formativo que conforma el nuestro programa universitario para mayores. Contemplamos la acción formativa TT como aquella actividad que, al igual que los trabajos fin de grado o de máster, marque la culminación de la trayectoria universitaria del alumno. Como en estos, bajo la supervisión de un tutor, se pretende que el alumno se enfrente a una tarea de complejidad superior a la desarrollada a lo largo de su paso por las aulas universitarias, invitándole a realizar un estudio bibliográfico, a aplicar los conocimientos adquiridos, a poner en juego las dotes de creatividad y originalidad, a obtener unos resultados y a evaluar cuantitativamente su esfuerzo. Los alumnos reciben una formación previa a través del Curso de Introducción a la Investigación en Ciencia y Tecnología, de carácter teórico-práctico, con una duración de 30 horas. En este curso, además de introducirlos en aspectos conceptuales de la investigación en estos ámbitos y su metodología, se aborda el uso de las nuevas tecnologías en las distintas etapas de la investigación: la actualización de la información, el tratamiento de los datos o la divulgación de los resultados obtenidos. El uso de bases de datos, el tratamiento de los datos a través del Microsoft Excel ${ }^{\mathrm{m}} \mathrm{o}$ la presentación de los resultados mediante el Microsoft Power Point ${ }^{\text {tm }}$ son algunas de las herramientas de las TIC que los alumnos aprenden a utilizar. La historia de la ciencia y la tecnología, las matemáticas, la astronomía o la biología, son algunos de los campos en los que nuestros alumnos han realizado тT. El alumno elabora una memoria final de su trabajo y un póster del mismo. Al final del curso se lleva a cabo una exposición de los pósteres de los trabajos realizados.

Palabras clave: programas universitarios para mayores, divulgación de la ciencia y la tecnología, Tic y personas mayores 


\section{Introducción}

En la Universidad Politécnica de Cartagena (UPCT) se creó en el curso académico 2003/2004 la Universidad de Mayores, con el objetivo fundamental de facilitar a las personas mayores su desarrollo personal y social, con el espíritu de establecer una justa correspondencia con lo que ellas, mediante su trabajo y esfuerzo, han aportado a nuestra sociedad, devolviéndoselo en forma de bienes culturales (UPCT, 2011a).

Los destinatarios de la Universidad de Mayores son personas mayores de 50 años y su plan de estudios está estructurado en tres cursos, divididos en dos cuatrimestres cada uno. En cada cuatrimestre, los alumnos han de realizar cuatro asignaturas. Todas las asignaturas tienen una carga lectiva de 30 horas, de las cuales 20 horas tienen carácter teórico y las 10 horas restantes son de carácter eminentemente práctico (UPCT, 2011b), en las que se incluyen actividades tales como: prácticas en laboratorio, en talleres en aula de informática, visitas, excursiones... Dado el perfil de nuestra universidad, científico-tecnológico y de gestión empresarial, la oferta formativa de la Universidad de Mayores se compone de un volumen alto de asignaturas relativas a estos ámbitos del conocimiento, suponiendo más del $60 \%$ del total de las asignaturas que conforman el plan de estudios, (UPCT, 2011c). Entre las asignaturas del campo de la ciencia, la tecnología o la gestión empresarial, que se ofertan destacamos: biología humana y salud, historia de la tecnología, contar bien para vivir mejor, patrimonio geológico, informática, flora ornamental y jardinería, medio ambiente, la relación con los bancos, astronomía, productos financieros... Las asignaturas son planteadas por los correspondientes equipos docentes buscando siempre la vertiente más amena y divulgativa de los contenidos que se presentan, suponiendo en tal caso un instrumento muy valioso las prácticas y actividades complementarias que puedan programarse.

Los alumnos que superan el plan de estudios, obtienen un diploma de la UPCT, que acredita el número total de créditos realizados, así como las materias cursadas.

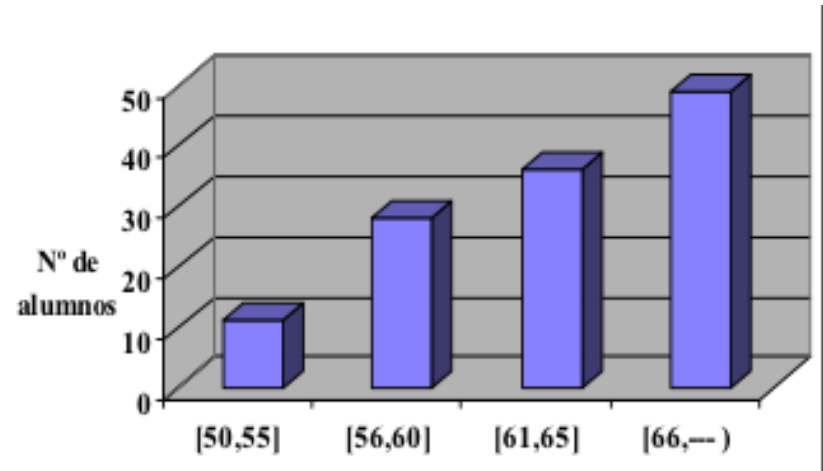

Figura 1. Distribución por edad de los alumnos de la Universidad de Mayores

Aquellos alumnos que han completado los tres cursos del plan de estudios de la Universidad de Mayores y que desean continuar con una formación permanente en nuestra institución pueden hacerlo a través de una doble vía: el aula permanente de mayores o los trabajos tutorizados. En el aula permanente de mayores, durante el Curso Académico se ofrecen distintos itinerarios formativos. En concreto, Los Huertos de Ocio, es una actividad que se desarrolla en la Estación Experimental Agroalimentaria Tomás Ferro, consistente en pequeñas parcelas de terreno destinadas al cultivo de productos típicos de la huerta mediante técnicas respetuosas con el medio ambiente. Esta actividad se organiza en dos semestres, coincidiendo con los ciclos de cultivo: otoño/invierno y primavera/verano. La otra acción formativa integrada en el aula permanente de mayores consiste en cursos de 20 horas de duración, cuya oferta en el presente curso académico contempla las siguientes materias (UPCT, 2011d): arqueología, apuntes de salud, introducción a los medios de comunicación y cultura de Cartagena y su entorno.

La segunda vía de formación permanente, la constituye los Trabajos Tutorizados (тт) cuyos objetivos, metodología docente utilizada y resultados obtenidos representan el núcleo del presente trabajo.

Para concluir, destacamos que, en el curso académico 2011/2012 un total de 460 personas mayores participan en las actividades programadas por la UPCT, dentro del proyecto educativo Universidad de Mayores, siendo interesante resaltar el dato de que la mayoría de su alumnado son mujeres ( $71 \%$ del total). 
En la figura 1 se muestra la distribución por intervalos de edad de los alumnos que participan en la Universidad de Mayores.

De la observación de estos dos resultados, se desprende que el perfil de alumno de la Universidad de Mayores es una mujer que supera los 60 años.

\section{Objetivos}

La acción formativa dirigida a personas mayores denominada trabajos tutorizados se implantó en la Universidad Politécnica de Cartagena en el curso académico 2008/2009, siendo sus destinatarios aquellos alumnos que han completado y superado los tres cursos del plan de estudios de la Universidad de Mayores (UPCT, 2011e). En nuestra universidad esta acción formativa TT se concibe como aquella actividad que, al igual que los trabajos fin de grado o de máster, marca la culminación de la trayectoria universitaria del alumno en la titulación cursada. Como en éstos, bajo la supervisión de un tutor, se pretende que el alumno se enfrente a una tarea de complejidad superior a la desarrollada a lo largo de su paso por las aulas universitarias, cuya elaboración requerirá, entre otros aspectos, los siguientes: aplicar los conocimientos adquiridos, integrar diversas disciplinas previamente cursadas, poner en juego sus dotes de creatividad y originalidad, obtener unos resultados y evaluar cuantitativamente su esfuerzo.

Para la realización de los trabajos tutorizados, los alumnos desarrollarán la siguiente línea de formación:

- Curso de Introducción a la Investigación en Ciencia y Tecnología, que formará al alumno en técnicas de investigación general, búsqueda de información y manejo de bases de datos, pautas para la elaboración de un trabajo de investigación, etc. a través de clases teóricoprácticas con una duración de 30 horas, que se ha programado del 19 de octubre al 21 de Diciembre del 2011.

- Realización de un trabajo bajo la supervisión de un tutor, dedicando a ello un tiempo estimado de 90 horas, que se desarrollará del 10 de enero al 20 de mayo del 2012.
Una vez que el alumno ha presentado y defendido el trabajo realizado, se le otorgará la correspondiente acreditación mediante un diploma de la UPCT, que no faculta para el ejercicio de una profesión (UPCT, 2011e).

\section{Metodología}

Como se ha indicado, los alumnos que participan en los trabajos tutorizados han de realizar previamente el Curso de Introducción a la Investigación en Ciencia y Tecnología, de carácter teórico-práctico, en el que se combina el aula con el laboratorio o el aula de informática. En este curso se abordan los siguientes contenidos:

1. Investigación en ciencia y tecnología. Aspectos conceptuales.

2. Estudio teórico de algunas investigaciones sencillas descritas en la bibliografía.

3. La actualización de la información.

- Búsqueda en Internet y en bases de datos.

4. El tratamiento de datos.

- El tratamiento informático de los datos. Introducción al uso de Microsoft Excel ${ }^{\mathrm{TM}} \mathrm{y}$ su aplicación al tratamiento de datos.

5. Divulgación de los resultados de las investigaciones científicas.

- Introducción al uso de programas informáticos (Microsoft Power Point ${ }^{\mathrm{Ti}}$ ).

- Presentación en forma de artículo científico, de comunicación oral o de póster.

Completadas las 30 horas formativas de este curso, el alumno comienza la elaboración de su trabajo en el ámbito de conocimiento y con el tutor elegidos al formalizar su matrícula. Los tutores de estos trabajos son profesores universitarios con una dilatada experiencia docente en el programa formativo Universidad de Mayores y, por tanto, con un conocimiento muy cercano del mismo, tanto sobre el enfoque que ha de imprimirse a estas disciplinas, como de los perfiles y características de este alumnado.

Entre las razones que pueden llevar a los alumnos a elegir un trabajo concreto, podríamos señalar: la preferencia por un área de conocimiento determi- 
nada, la inquietud y el interés personales por uno de los temas ofertados, la aptitud hacia una materia concreta o la profundización en los contenidos de alguna de las disciplinas cursadas.

La astronomía, el medio ambiente, el consumo, las matemáticas, los materiales, la ingeniería, la historia de la ciencia y la cultura clásica son los ámbitos de conocimiento en las que se centra la oferta de los TT en el curso académico 2011/2012, ofreciéndose a los alumnos 21 plazas (UPCT, 2011f).

Cada tutor, de acuerdo con su alumno o alumnos, fijará un horario semanal para llevar a cabo la labor. Así, cada semana se programa una sesión de una hora y treinta minutos de duración, en la que el alumno y su tutor se reunirán para desarrollar, de forma secuencial, aspectos tales como:

- Indicaciones sobre el ámbito general del tema de trabajo elegido, las fuentes bibliográficas fundamentales de consulta o las posibles líneas del trabajo.

- Planificación y programación temporal de las distintas etapas en la elaboración del Trabajo.

- Orientación al alumno en el desarrollo de cada una de las anteriores etapas y resolución de aquellas dudas o dificultades que le puedan surgir al alumno.

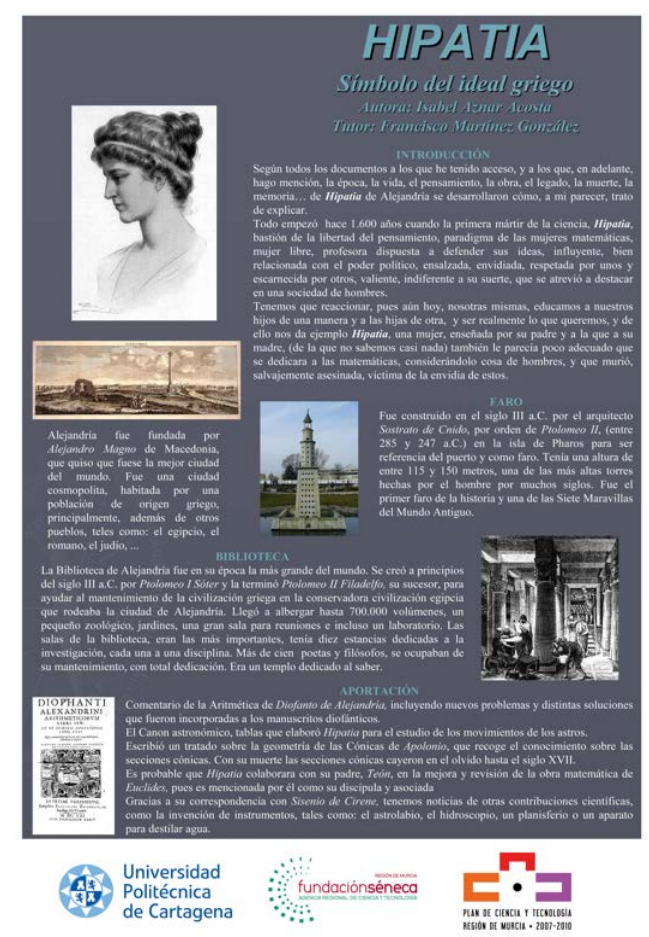

Figura 3a
Tabla 1. Distribución de los alumnos por ámbitos de conocimiento

\begin{tabular}{|l|r|}
\hline Área de conocimiento & $\begin{array}{l}\text { N. } \\
\text { alumnos }\end{array}$ \\
\hline Biología humana y salud & 1 \\
\hline Medio ambiente & 3 \\
\hline Historia de la ciencia y la técnica & 6 \\
\hline Astronomía & 12 \\
\hline Ciencia y tecnología & 7 \\
\hline Matemáticas & 12 \\
\hline Cultura clásica & 8 \\
\hline
\end{tabular}

- Evaluación del grado de consecución de las metas marcadas, inicialmente, para cada una de las etapas programadas en el trabajo, disponiéndose así de un criterio objetivo para ir completando las etapas o profundizar sobre un extremo concreto o volver a plantearse algunas de estas metas iniciales.

- Supervisión de los contenidos que se recogerán en cada uno de los apartados, que van a conformar la memoria final del trabajo.

- Apoyo técnico al alumno, si es preciso, para la elaboración de la memoria final del trabajo, su

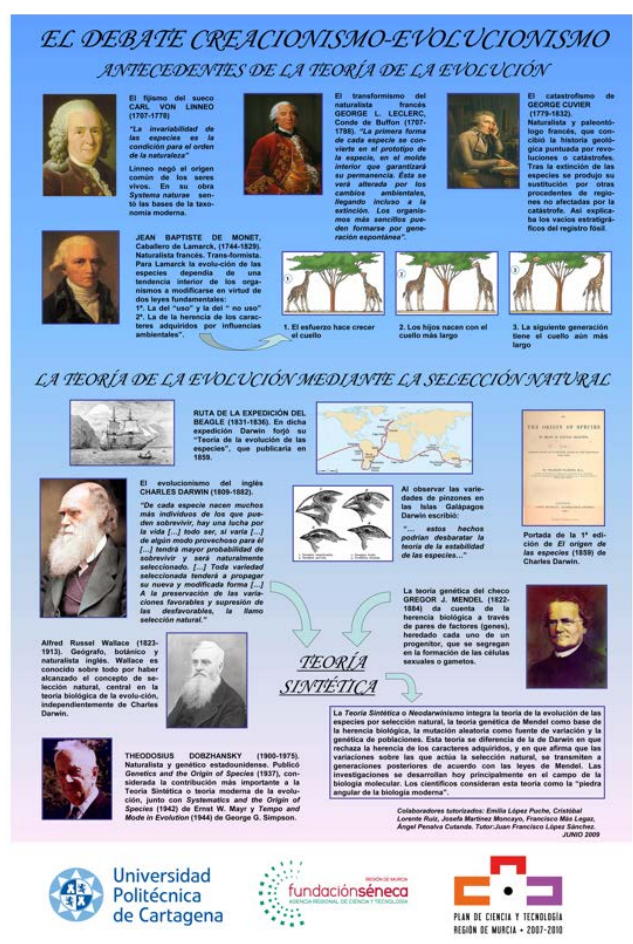

Figura 3b 
presentación oral y la realización de un póster de la misma

Una vez terminada la memoria final del trabajo, el alumno la entregará a su tutor, junto con un póster descriptivo del mismo. La Universidad de Mayores, en la primera quincena de junio, programará una fecha para la presentación oral del trabajo por parte del alumno. Así mismo, en estas fechas, se llevará a cabo una Exposición en la que se muestran los pósteres elaborados por los alumnos en el curso académico. En los tres últimos cursos académicos esta exposición se planificó en los espacios expositivos de la Residencia Universitaria Alberto Colao. Coincidiendo con la inauguración de la citada exposición, se programó un acto académico, en el que se entregó a los alumnos un diploma de la Universidad Politécnica de Cartagena, que acredita la actividad.

\section{Resultados}

Desde el curso académico 2008/2009 han participado 49 alumnos en el proyecto pormativo Trabajos Tutorizados, cuya distribución por sexo es la siguiente: el $59 \%$ son mujeres y el restante $41 \%$ hombres.

En la tabla 1 se muestra la distribución de los alumnos según las áreas de conocimiento en las que se han elaborado los TT.

En las figuras $3 a$ y $3 b$, se muestran dos de los pósteres realizados por los alumnos del proyecto formativo Trabajos Tutorizados, correspondientes al curso académico 2008/2009. La figura $3 a$ recoge el póster sobre Hipatia de Alejandría correspondiente al área de conocimiento matemáticas. En la figura $3 b$ se muestra el trabajo titulado El debate creacionismo-revolucionismo, correspondiente al área historia de la ciencia y la técnica.

Comentar, por último, que la colección de pósteres correspondientes a los TT realizados hasta la fecha ha sido objeto de exposición en diferentes eventos relacionados con la divulgación de la ciencia y la tecnología o con el ámbito de las personas mayores, tanto en nuestra universidad como en otras instituciones.

\section{Referencias}

UPCт. Universidad Politécnica de Cartagena (2011a). Normativa de la Universidad de Mayores de la UPCT. Curso Académico 2011/12

upct Universidad Politécnica de Cartagena (2011b). Horario de la Universidad de Mayores de la upct. Curso Académico 2011/13

UPCT. Universidad Politécnica de Cartagena (2011c). Resúmenes de las asignaturas de la Universidad de Mayores de la UPCT

UPCт. Universidad Politécnica de Cartagena (2011d). Aula Permanente de Mayores. Curso académico 2011/2012

UPCT. Universidad Politécnica de Cartagena (2011e). Normativa de los Trabajos Autorizados de la Universidad de Mayores de la UPCT. Curso académico 2011/2012

UPCT. Universidad Politécnica de Cartagena (2011f). Introducción a la Investigación en Ciencia y Tecnología. 